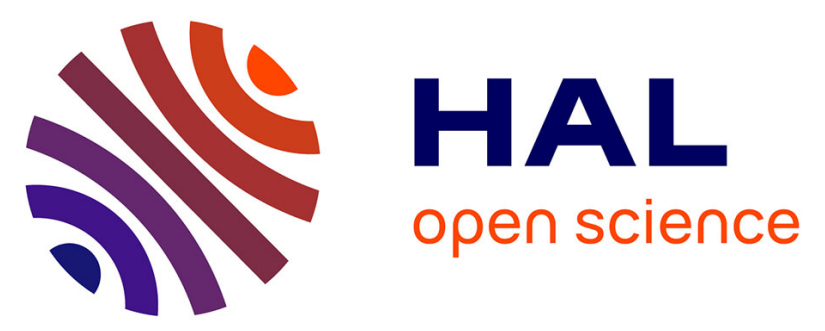

\title{
Field and angular dependence of the Sommerfeld coefficient in Al-doped MgB2 single crystals
}

Z. Pribulova, Thierry Klein, Jacques Marcus, C. Marcenat, M. S. Park, H.S. Lee, H.G. Lee, S.I. Lee

\section{- To cite this version:}

Z. Pribulova, Thierry Klein, Jacques Marcus, C. Marcenat, M. S. Park, et al.. Field and angular dependence of the Sommerfeld coefficient in Al-doped MgB2 single crystals. Physical Review B: Condensed Matter and Materials Physics (1998-2015), 2007, 76, pp.180502(R). 10.1103/PhysRevB.76.180502 . hal-00957174

\section{HAL Id: hal-00957174 \\ https://hal.science/hal-00957174}

Submitted on 10 Mar 2014

HAL is a multi-disciplinary open access archive for the deposit and dissemination of scientific research documents, whether they are published or not. The documents may come from teaching and research institutions in France or abroad, or from public or private research centers.
L'archive ouverte pluridisciplinaire HAL, est destinée au dépôt et à la diffusion de documents scientifiques de niveau recherche, publiés ou non, émanant des établissements d'enseignement et de recherche français ou étrangers, des laboratoires publics ou privés. 


\title{
Field and angular dependence of the Sommerfeld coefficient in Al-doped $\mathrm{MgB}_{2}$ single crystals
}

\author{
Z. Pribulova, ${ }^{1,2, *}$ T. Klein, ${ }^{1,2}$ J. Marcus, ${ }^{1}$ C. Marcenat,${ }^{3}$ M. S. Park, ${ }^{4}$ H.-S. Lee,${ }^{4}$ H.-G. Lee,${ }^{4}$ and S.-I. Lee ${ }^{4}$ \\ ${ }^{1}$ Institut Néel, CNRS, F-38042 Grenoble Cedex 9, France \\ ${ }^{2}$ Université Joseph Fourier, F-38041 Grenoble, France \\ ${ }^{3}$ CEA-Grenoble, Département de Recherche Fondamentale sur la Matière Condensée, F-38054 Grenoble, France \\ ${ }^{4}$ NVCRICS and Department of Physics, Pohang University, Pohang 790-784, Republic of Korea
}

(Received 9 July 2007; published 7 November 2007)

\begin{abstract}
The angular and field dependence of the Sommerfeld coefficient $\gamma=\lim C_{e l} /\left.T\right|_{T \rightarrow 0}\left(C_{e l}\right.$ being the electronic contribution to the specific heat) has been measured in Al-doped $\mathrm{MgB}_{2}$ single crystals (for $x=0, x=0.1$, and $x \sim 0.2$ ). We show that the decomposition previously introduced to describe $\gamma(H, \theta)$ (where $\theta$ is the angle between the applied field and the $c$ axis) in pure samples [Phys. Rev. Lett. 98, 137001 (2007)] is well adapted to doped samples: e.g., the contribution of the $\sigma$ band to the specific heat is proportional to $B / B_{c 2}(\theta)$ whereas the contribution of the $\pi$ band is isotropic but highly nonlinear in field. We hence present the evolution of the coherence lengths of the two bands and corresponding Fermi velocities with doping.
\end{abstract}

DOI: $10.1103 /$ PhysRevB.76.180502

PACS number(s): 74.50.+r, 74.25.Op, 74.72. $-\mathrm{h}$

Since the discovery of its superconductivity, $\mathrm{MgB}_{2}$ has been the focus of a large amount of both theoretical and experimental works. Besides its high critical temperature $\left(T_{c} \sim 40 \mathrm{~K}\right), \mathrm{MgB}_{2}$ is a unique example of a system in which two weakly coupled bands with very different characters coexist (an almost isotropic $\pi$ band and a quasi-twodimensional $\sigma$ band). As the high critical temperature is a direct consequence of the very efficient coupling of the holes on the top of the $\sigma$ band with $E_{2 g}$ phonon modes, many experiments have then been devoted to the influence of electronic doping on the physical properties of this system. Both carbon- $\left[\mathrm{Mg}\left(\mathrm{B}_{1-x} \mathrm{C}_{x}\right)_{2}\right]$ (Refs. 1-3) and aluminum$\left(\mathrm{Mg}_{1-x} \mathrm{Al}_{x} \mathrm{~B}_{2}\right)$ (Refs. 4-8) doped samples have been investigated. A decrease of the critical temperature with $x$ has been observed in both cases; however, whereas all studies agree on a significant increase of the upper critical fields for both $H \| c$ and $H \| a b$ due to strong impurity scattering in C-doped samples, ${ }^{1-3}$ most of the studies now also agree that those fields decrease in $\mathrm{Mg}_{1-x} \mathrm{Al}_{x} \mathrm{~B}_{2}$.

We have shown in Ref. 9 that the anisotropy $\Gamma$ of the pure system is strongly field dependent and rapidly increases with field above $\sim 0.3 \mathrm{~T}$ due to a shrinking of the $\pi$-band coherence length $\left(\xi_{\pi}\right)$, reaching $\xi_{\sigma}^{a b}$ for $B=B_{c 2}^{c}$. The aim of the present work is to present a systematic study of the field dependence of those fundamental parameters (coherence length and anisotropy) with electronic doping in Al-doped samples. Therefore we have performed a detailed analysis of the angular and field dependence of the Sommerfeld coefficient $\gamma=\lim C_{e l} /\left.T\right|_{T \rightarrow 0}\left(C_{e l}\right.$ being the electronic contribution to the specific heat) in Al-doped magnesium diboride single crystals (for $x=0, x=0.1$, and $x \sim 0.2$ ). As discussed in Ref. 8 , the nominal composition is not perfectly well defined for the highest doping content (this sample is hence referred to as $x$ "on the order of" 0.2 and not $x=0.2$ ). Second phase precipitation cannot be fully excluded for those high Al contents [i.e., for $x>0.1$ (Ref. 10)], but specific heat measurements are very sensitive to the sample homogeneity and all measured samples present well-defined specific heat jumps at $T_{c .}{ }^{8}$ More details on the experimental procedure and ac technique used here are given in Refs. 8 and 9.
As previously observed in pure samples, we show that the contribution of the $\sigma$ band to the specific heat follows a classical behavior being proportional to $B / B_{c 2}(\theta)$ (where $\theta$ is the angle between the applied field and the $c$ axis) whereas the contribution of the $\pi$ band is isotropic but highly nonlinear in field. We hence obtained the evolution of the coherence lengths (and corresponding Fermi velocities) of the $\sigma$ and $\pi$ bands with doping.

For low values of the applied field $H_{a}$ (up to a few firstpenetration fields), the $C_{p}$ measurements are strongly hysteretic due to very different vortex distributions in the sample on the ascending and descending branches of the field cycle. ${ }^{9,11}$ In order to obtain the evolution of the Sommerfeld coefficient at low field we have thus determined the average induction $\bar{B}$ (hereafter denoted $B$ ) at the surface of sample as a function of $H_{a}$ using a miniature Hall probe array ${ }^{12}$ for both $H \| c$ and $H \| a b$ (see Ref. 9 for details). The $B$ dependence of the Sommerfeld coefficient is displayed in Fig. 1 for $x=0$ and $x \sim 0.2$. As shown, at low $B, \gamma$ is isotropic and, as expected for classical (dirty ${ }^{13}$ ) superconductors, varies linearly. However, this low-field linear regime is only visible on a very restricted field range (up to $B \sim 0.1 \mathrm{~T}$ and $B \sim 0.2 \mathrm{~T}$ for $x=0$ and $x \sim 0.2$, respectively) and $\gamma$ rapidly becomes highly nonlinear and finally anisotropic for $B \geqslant 0.3 \mathrm{~T}$ and $B \geqslant 0.5 \mathrm{~T}$ for $x=0$ and $x \sim 0.2$, respectively. Note that the very strong nonlinearity observed for $\gamma / \gamma_{N} \geqslant 0.4$ for $x=0$ is much weaker for $x \sim 0.2$ and sets in only for $\gamma / \gamma_{N} \geqslant 0.6$, reflecting the decrease of the contribution of the $\sigma$ band with doping (see below for details).

This nonlinear behavior has initially ${ }^{14}$ been qualitatively explained by writing $\gamma=\omega \gamma_{\pi}+(1-\omega) \gamma_{\sigma}$ where $\omega$ is the relative weight of the $\pi$ band [on the order of $1 / 2$ (Ref. 15)] and assuming that the contribution of each band is linear for $B$ $<B_{i}=\Phi_{0} / 2 \pi \xi_{i}^{2}$ (with $i=\pi$ or $\sigma$ ). As the $\pi$ band is expected to be much more sensitive to $B$ than the $\sigma$ band, one hence gets two linear behaviors corresponding to $B<B_{\pi} \ll B_{c 2}$ and $B>B_{\pi}$, respectively. However, we have shown that, even though a high-field linearlike regime can be observed for $B$ $>B_{c 2}^{c}=3 \mathrm{~T}$ (Ref. 9) (and hence $\theta \neq 0$ ), $\gamma$ can be better described by a $\left(B / B_{c 2}^{c}\right)^{\alpha}$ law on almost the complete field range 


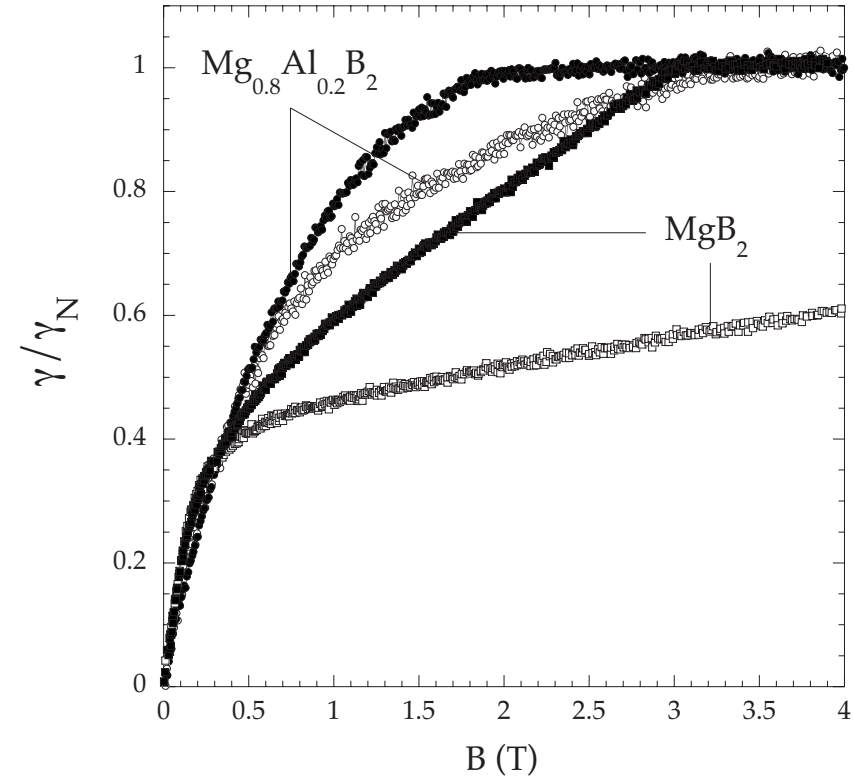

FIG. 1. Magnetic field dependence of the Sommerfeld coefficient $\gamma$ at $T=2.5 \mathrm{~K}$ for $H \| c$ (solid symbols) and $H \| a b$ (open symbols) in $\mathrm{MgB}_{2}$ (squares) and $\mathrm{Mg}_{0.8} \mathrm{Al}_{0.2} \mathrm{~B}_{2}$ (circles).

(with $\alpha \sim 0.4-0.5)$ for $H \| c$. Note also that for $H \| c$ the linearlike behavior at high field would extrapolate towards $\omega$ $\sim 0.35$ (for $B=0$ ) - i.e., much lower than the theoretical value on the order of 0.5 (see Fig. 1). The absence of this high-field linear regime for $\theta=0$ becomes even more obvious for $x \neq 0$ (see solid circles in Fig. 1 for $x \sim 0.2$ ). In order to get a better description of $\gamma(B, \theta)$, we have thus measured the angular dependence of the Sommerfeld coefficient for various applied fields [see Fig. 2(a) for $x=0$ at $T=2 \mathrm{~K}$ ].

In classical superconductors, $\gamma$ is expected to depend on $B / B_{c 2}(\theta)$ where $B_{c 2}(\theta)=B_{c 2}^{a b} / \sqrt{\sin ^{2} \theta+\Gamma_{B_{c 2}}^{2} \cos ^{2} \theta} \cdot{ }^{16}$ Such a dependence obviously does not hold in $\mathrm{MgB}_{2}$ but $\gamma(B, \theta)$ can still be described as $f\left(B / B_{c 2}^{*}\right)$, introducing a field-dependent $\Gamma(B)$ value through $B_{c 2}^{*}=B_{c 2}^{a b} / \sqrt{\sin ^{2} \theta+\Gamma(B)^{2} \cos ^{2} \theta}$. The corresponding $\Gamma(B)$ values have been reported in the inset of Fig. 3 for the three $x$ values. As shown in Ref. 9, $\Gamma$ is on the order of 1 at low field (i.e., $\sim \Gamma_{B_{c 1}}$ ) and then sharply increases, reaching $\Gamma_{B_{c 2}}$ for $B \geqslant B_{c 2}^{c}$, indicating that no superconductivity is obtained in the $\pi$ band above this field. More precisely, we have shown in Ref. 9 that, in pure $\mathrm{MgB}_{2}$, the angular dependence of $\gamma$ can actually be written as

$$
\frac{\gamma(B, \theta)}{\gamma_{N}}=\omega \frac{B}{B_{\pi}(B)}+(1-\omega) \frac{B}{B_{c 2}(\theta)},
$$

where $B_{\pi}$ is a field-dependent parameter introduced to describe the highly nonlinear (but isotropic) contribution of the $\pi$ band. The contribution of the $\sigma$ band, $\gamma_{\sigma}$ [second term in Eq. (1)], hence varies linearly with $B$ and its anisotropy is fully determined by the one of $B_{c 2}(\theta)$ through the $B / B_{c 2}(\theta)$ ratio. Following Eq. (1), the ratio

$$
R=\frac{\gamma(B, \theta)-\gamma(B, 90)}{\gamma_{N} b_{c 2}^{a b}}=(1-\omega)\left[\sqrt{\sin ^{2} \theta+\Gamma_{B_{c 2}}^{2} \cos ^{2} \theta}-1\right]
$$

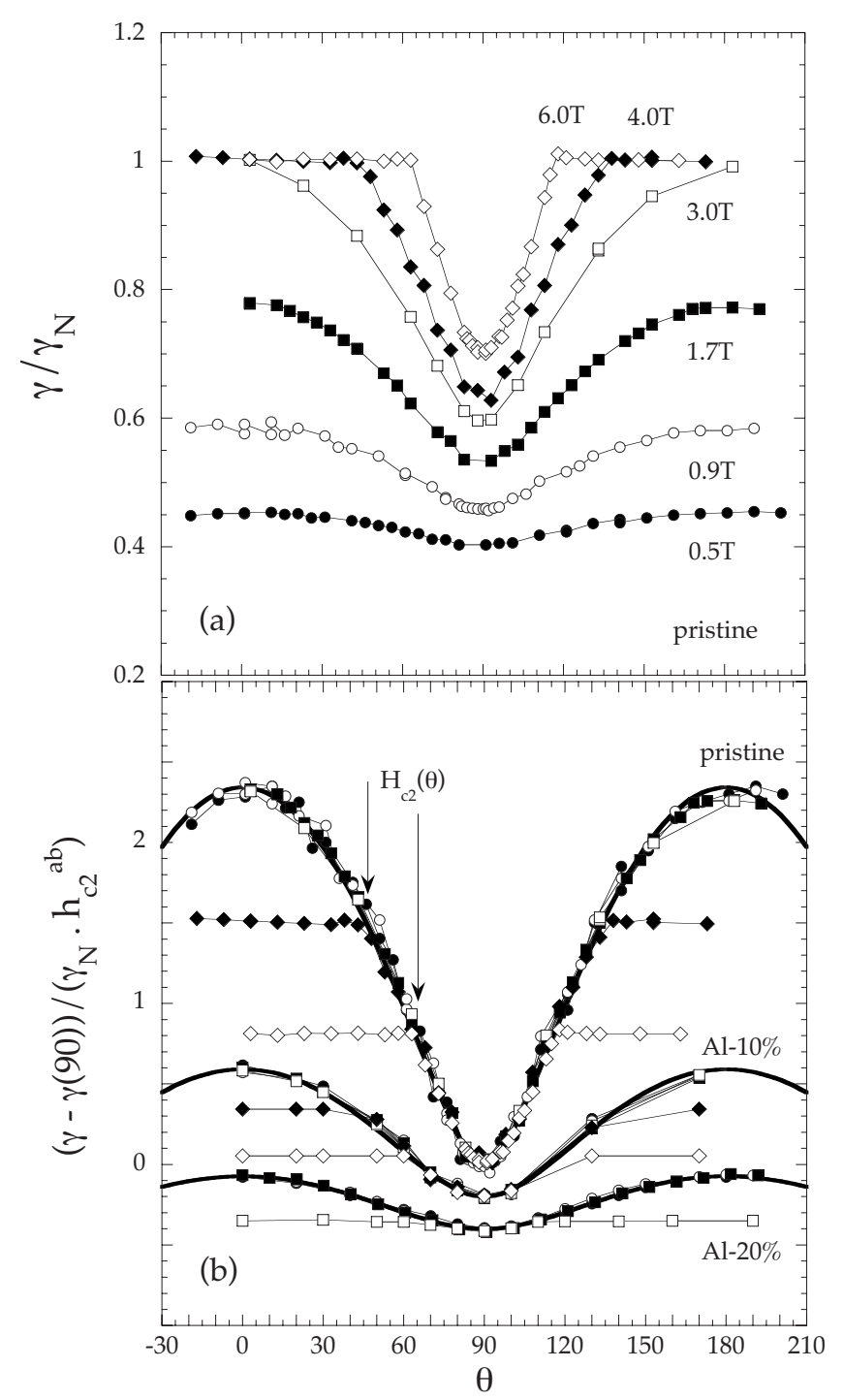

FIG. 2. (a) Angular dependence of the Sommerfeld coefficient for the indicated field values in a pure $\mathrm{MgB}_{2}$ single crystal. (b) $[\gamma(B, \theta)-\gamma(B, \theta=90)] / \gamma_{N} \times B_{c 2}^{a b} / B$ as a function of $\theta$ for $x=0$ (open diamonds, 6.0 T; solid diamonds, $4.0 \mathrm{~T}$; open squares, $3.0 \mathrm{~T}$; solid squares, $1.7 \mathrm{~T}$; open circles, $0.9 \mathrm{~T}$; solid circles, $0.5 \mathrm{~T}$ ), $x=0.1$ (open diamonds, 4.0 T; solid diamonds, 3.0 T; open squares, $2.5 \mathrm{~T}$; solid squares, $2.0 \mathrm{~T}$; open circles, $1.5 \mathrm{~T}$; solid circles, $1.0 \mathrm{~T}$ ), and $x \sim 0.2$ (open diamonds, $3.0 \mathrm{~T}$; solid diamonds, $1.5 \mathrm{~T}$; open squares, $1.0 \mathrm{~T}$; solid squares, $0.5 \mathrm{~T}$ ). The data for $x=0.1$ and $x$ $\sim 0.2$ have been shifted along the vertical axis for clarity. The solid lines are fits to the data using Eq. (2) (see text for details).

$\Gamma_{B_{c 2}}$ being now the field-independent anisotropy of the upper critical field. ${ }^{17}$

As shown in Fig. 2(b), all the $\gamma(\theta, B)$ data collapse onto single curves for all three $x$ values when plotted as $R$ vs $\theta$, clearly showing that Eq. (1) describes the field and angular dependence of $\gamma$ for any doping level. The solid lines in Fig. 2(b) are fits to the data using Eq. (2). The $\Gamma_{B_{c 2}}$ values have been deduced from $B_{c 2}$ measurements, ${ }^{8}$ and the only free parameter was hence the weight of the $\sigma$ band, $\omega_{\sigma}=1-\omega$. As expected for electronic doping, $\omega_{\sigma}$ is decreasing with doping from $\sim 0.5$ for $x=0$ to $\sim 0.35-0.4$ for $x \sim 0.2$, in excellent 


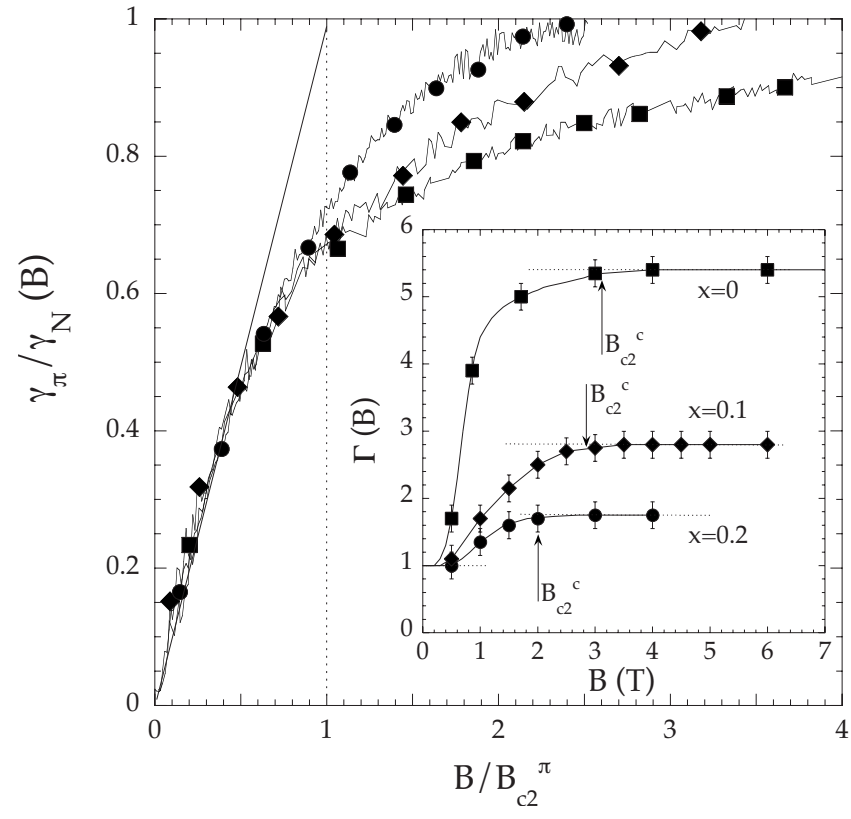

FIG. 3. Field dependence of the contribution of the $\pi$ band to the Sommerfeld coefficient in $\mathrm{Al}_{x} \mathrm{Mg}_{1-x} \mathrm{~B}_{2}$ for $x=0$ (squares), $x$ $=0.1$ (diamonds), and $x \sim 0.2$ (circles). Inset: field dependence of the effective anisotropy ratio (see text for details) for the corresponding samples.

agreement with $\omega_{\sigma}$ values deduced from the temperature dependence of the zero-field specific heat ${ }^{18}$ (see also Ref. 7).

As $\gamma_{\sigma}$ varies linearly with $B$, the contribution of the $\pi$ band can be obtained through $\gamma_{\pi} / \gamma_{N}=\left[\gamma / \gamma_{N}-(1\right.$ $\left.-\omega) B / B_{c 2}\right] / \omega$. The corresponding $\gamma_{\pi} / \gamma_{N}$ values are displayed in Fig. 3 as a function of $B / B_{c 2}^{\pi}$ where $B_{c 2}^{\pi}$ $=\left.\lim B_{\pi}(B)\right|_{B \rightarrow 0} . B_{c 2}^{\pi}$ increases from $\sim 0.25 \mathrm{~T}$ for $x=0$ to $\sim 0.6 \mathrm{~T}$ for $x \sim 0.2$. However, as discussed in Ref. 9, superconductivity still survives in the $\pi$ band for $B>B_{c 2}^{\pi}$ due to the coupling with the $\sigma$ band, leading to a shrinking of the vortex core from $\xi^{\pi}(0)=\sqrt{\Phi_{0} / 2 \pi B_{c 2}^{\pi}}$ at low field down to $\xi^{\pi}\left(B \sim B_{c 2}^{c}\right)=\xi_{a b}^{\sigma}$. Since the $\pi$ band is isotropic, it can have only one $\theta$-independent $B_{c 2}$ value and superconductivity is hence destroyed in this band in all directions for $B \geqslant B_{c 2}^{c}$.

The $\sigma$-band coherence length can be deduced from the upper critical field, writing $B_{c 2}^{c}=\Phi_{0}^{2} /\left(2 \pi \xi_{a b}^{\sigma 2}\right)$ and $B_{c 2}^{a b}$ $=\Phi_{0}^{2} /\left(2 \pi \xi_{c}^{\sigma} \xi_{a b}^{\sigma}\right)$. The evolution of $\xi^{\sigma}$ with doping is displayed in Fig. 4(a), taking the $B_{c 2}$ values previously measured in Ref. 8. The corresponding Fermi velocities [solid symbols in Fig. 4(b)] can then be estimated using the Bardeen-Cooper-Schrieffer (BCS) single-band formula $\hbar v_{F}^{\sigma}$ $=\pi \Delta_{\sigma} \xi^{\sigma}$. For $x=0$, one obtains $v_{F, a b}^{\sigma} \sim 3.5 \times 10^{5} \mathrm{~m} / \mathrm{s}$ and $v_{F, c}^{\sigma} \sim 0.7 \times 10^{5} \mathrm{~m} / \mathrm{s}$ (taking $\Delta_{\sigma} \sim 7.1 \mathrm{meV}$ ), in fairly good agreement with band structure calculations ${ }^{19}$ [open symbols in Fig. 4(b)]. Note that the $\pi$-band Fermi velocity deduced from this BCS formula $\left(v_{F}^{\pi}=\pi \Delta_{\pi} \xi^{\pi} / \hbar \sim 4 \times 10^{5} \mathrm{~m} / \mathrm{s}\right.$ for $x$ $=0$ ) is also surprisingly close to the theoretical value (taking $\Delta_{\pi} \sim 2.4 \mathrm{meV}$ ). Indeed, as discussed in Ref. 9, GinzburgLandau calculations for two-gap superconductors rather suggested that $\xi^{\pi} / \xi_{a b}^{\sigma} \leqslant 2$ for reasonable parameters ${ }^{20}$ and the origin of this agreement hence still has to be understood.

The evolution of $v_{F}^{\sigma}$ with aluminum doping is displayed in

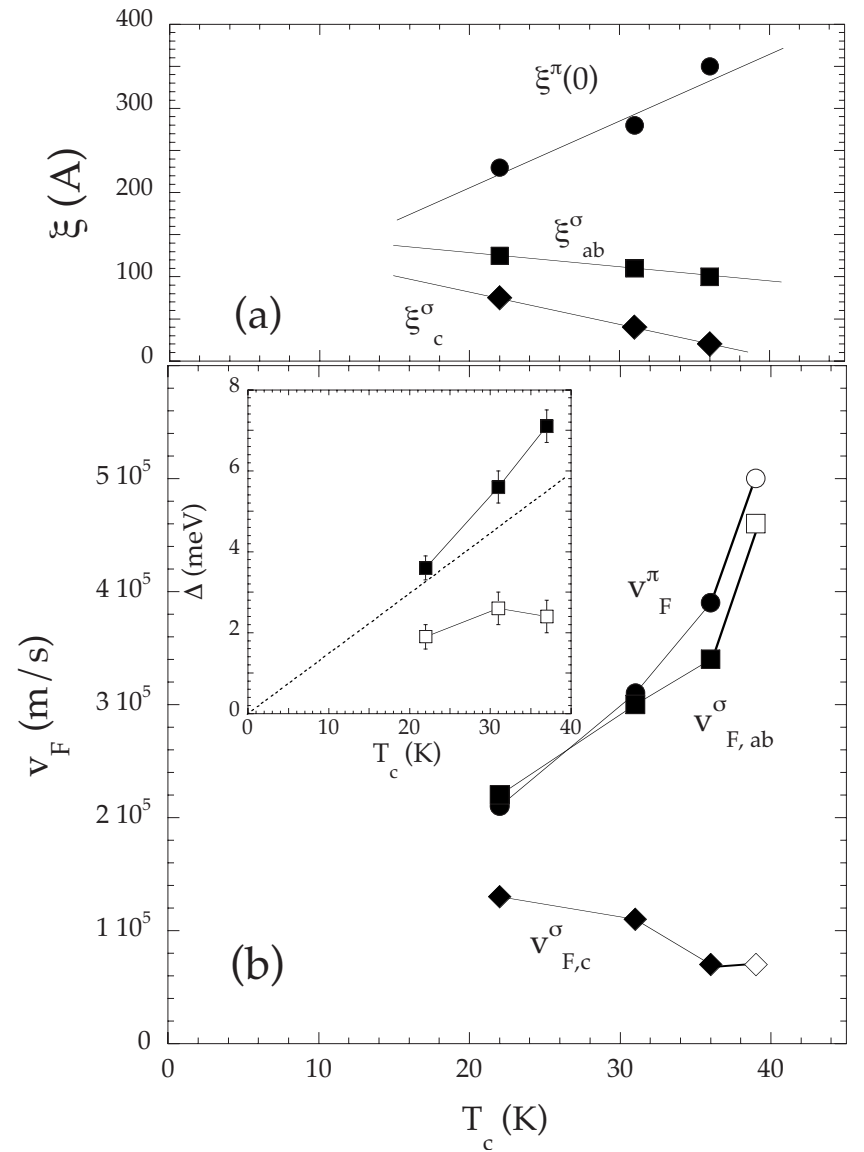

FIG. 4. (a) Evolution of the coherence length of the two bands with doping. (b) Corresponding Fermi velocities (solid circles) deduced from the standard BCS formula $v_{F}=\pi \Delta \xi / \hbar$, introducing the gap values displayed in the inset (solid symbols, $\Delta_{\sigma}$; open symbols, $\Delta_{\pi}$ ). The open symbols of the main panel are average theoretical values deduced from band structure calculations.

Fig. 4(a) [taking the gap values deduced from the temperature dependence of $C_{p}$; see inset of Fig. 4(b) and Ref. 8 for details]. Although in qualitative agreement with Putty et al., ${ }^{5}$ this evolution is much steeper than the one obtained by these authors from band structure calculations. Those calculations indeed suggested a $\sim 20 \%$ decrease of $v_{F, a b}^{\sigma}$ for $x$ varying from 0 to 0.2 (and a corresponding $\sim 20 \%$ increase in $v_{F, c}^{\sigma}$ ) whereas our data show that $v_{F, a b}^{\sigma}$ decreases by a factor of $\sim 2$ (and $v_{F, c}^{\sigma}$ increases by the same factor). The decrease of $\xi^{\pi}(0)$ with doping suggests that the $\pi$-band Fermi velocity also decreases by a factor of $\sim 2$ for $x \rightarrow 0.2$ [see Fig. 4(b)]. However, it is worth noting that $\xi^{\pi}(0)$ (and hence $v_{F}^{\pi}$ ) has been deduced from $B_{c 2}^{\pi}$ assuming that this band is clean whereas Putti et al. ${ }^{21}$ actually suggested that aluminum doping may lead to a significant increase of the $\pi$-band scattering rate. In a dirty limit, the decrease of the effective Fermi velocity reported in Fig. 4(b) would then correspond to a decrease of the diffusivity $D\left[V_{F, e f f}=(\pi \Delta / \hbar) \sqrt{\Phi_{0} / 2 \pi B_{c 2}} \propto \sqrt{\Delta D / \hbar}\right]$.

We have shown that the $\gamma(B, \theta) / \gamma_{N}=\omega B / B_{\pi}(B)+(1$ $-\omega) B / B_{c 2}(\theta)$ (where $B_{\pi}$ is a field-independent coefficient 
reflecting the shrinking of the $\pi$-band coherence length for $B>B_{c 2}^{\pi}$ ) decomposition of the Sommerfeld coefficient introduced in pure samples can be generalized to Al-doped $\mathrm{MgB}_{2}$ samples. Correspondingly, all $\xi$ values $\left(\xi^{\pi}\right.$ and $\left.\xi^{\sigma}\right)$ seem to converge towards a single value on the order of $150 \AA$ for high doping and a corresponding isotropic Fermi velocity $\sim 1.5 \times 10^{5} \mathrm{~m} / \mathrm{s}$.
We would like to thank S. Tajima and S. Lee for providing us some of the pristine samples. Z.P. thanks the Slovak Science and Technology Agency for partial support, Contracts Nos. APVT-51-016604 and LPP-0101-06. S.-I.L. acknowledges support by the Korea Science and Engineering Foundation through the Creative Research Initiative Program.
*Present address: Centre of Low Temperature Physics IEP SAS \& FS UPJS, Watsonova 47, SK-04001 Kosice, Slovakia.

${ }^{1}$ R. R. Ribeiro, S. L. Budko, C. Petrovic, and P. C. Canfield, Physica C 384, 227 (2003).

${ }^{2}$ Z. Hol'anová, J. Kačmarčík, P. Szabó, P. Samuely, I. Sheikin, R. A. Ribeiro, S. L. Bud'ko, and P. C. Canfield, Physica C 404, 195 (2004)

${ }^{3}$ R. H. T. Wilke, S. L. Bud'ko, P. C. Canfield, D. K. Finnemore, R. J. Suplinskas, and S. T. Hannahs, Phys. Rev. Lett. 92, 217003 (2004).

${ }^{4}$ M. Putti, M. Affronte, P. Manfrinetti, and A. Palenzona, Phys. Rev. B 68, 094514 (2003).

${ }^{5}$ M. Putti, C. Ferdeghini, M. Monni, I. Pallecchi, C. Tarantini, P. Manfrinetti, A. Palenzona, D. Daghero, R. S. Gonnelli, and V. A. Stepanov, Phys. Rev. B 71, 144505 (2005).

${ }^{6}$ M. Angst, S. L. Bud'ko, R. H. T. Wilke, and P. C. Canfield, Phys. Rev. B 71, 144512 (2005).

${ }^{7}$ L. D. Cooley, A. J. Zambano, A. R. Moodenbaugh, R. F. Klie, Jin-Cheng Zheng, and Yimei Zhu, Phys. Rev. Lett. 95, 267002 (2005).

${ }^{8}$ T. Klein, L. Lyard, J. Marcus, C. Marcenat, P. Szabo, Z. Holanova, P. Samuely, B. W. Kang, H.-J. Kim, H.-S. Lee, H.-K. Lee, and S.-I. Lee, Phys. Rev. B 73, 224528 (2006).

${ }^{9}$ Z. Pribulova, T. Klein, J. Marcus, C. Marcenat, F. Levy, M. S. Park, H. G. Lee, B. W. Kang, S. I. Lee, S. Tajima, and S. Lee, Phys. Rev. Lett. 98, 137001 (2007).

${ }^{10}$ J. Karpinski, N. D. Zhigadlo, G. Schuck, S. M. Kazakov, B. Batlogg, K. Rogacki, R. Puzniak, J. Jun, E. Müller, P. Wägli, R. Gonnelli, D. Daghero, G. A. Ummarino, and V. A. Stepanov, Phys. Rev. B 71, 174506 (2005).

${ }^{11}$ L. Lyard, T. Klein, J. Marcus, R. Brusetti, C. Marcenat, M. Konczykowski, V. Mosser, K. H. Kim, B. W. Kang, H. S. Lee, and S. I. Lee, Phys. Rev. B 70, 180504(R) (2004).

${ }^{12}$ The induction $B$ at the surface of the sample has been averaged over $\sim 25$ points for $H \| c$ and $\sim 10$ points for $H \| a b$.

${ }^{13}$ Deviations from this linear behavior are expected in very clean systems: see V. G. Kogan, Phys. Rev. B 66, 020509(R) (2002); V. G. Kogan and N. V. Zhelezina, ibid. 69, 132506 (2004).

${ }^{14}$ F. Bouquet, Y. Wang, I. Sheikin, T. Plackowski, A. Junod, S. Lee, and S. Tajima, Phys. Rev. Lett. 89, 257001 (2002).

${ }^{15}$ A. Y. Liu, I. I. Mazin, and J. Kortus, Phys. Rev. Lett. 87, 087005 (2001); A. A. Golubov, J. Kortus, O. V. Dolgov, O. Jepsen, Y. Kong, O. K. Andersen, B. J. Gibson, K. Ahn, and R. K. Kremer, J. Phys.: Condens. Matter 14, 1353 (2002); H. J. Choi, D. Roundy, H. Su, M. L. Cohen, and S. G. Louie, Nature (London) 418, 758 (2002); I. I. Mazin, O. K. Andersen, O. Jepsen, O. V. Dolgov, J. Kortus, A. A. Golubov, A. B. Kuz'menko, and D. van der Marel, Phys. Rev. Lett. 89, 107002 (2002).

${ }^{16}$ Small deviations from the $B_{c 2}=B_{c 2}^{a b} / \sqrt{\sin ^{2} \theta+\Gamma^{2} \cos ^{2} \theta}$ law may be expected in two gap superconductors but those deviations are extremely small at low temperature: A. Rydh, U. Welp, A. E. Koshelev, W. K. Kwok, G. W. Crabtree, R. Brusetti, L. Lyard, T. Klein, C. Marcenat, B. Kang, K. H. Kim, K. H. P. Kim, H.-S. Lee, and S.-I. Lee, Phys. Rev. B 70, 132503 (2004).

${ }^{17}$ As expected, $[\gamma(B, \theta)-\gamma(B, \theta=90)] / \gamma_{N} \times B_{c 2}^{a b} / B$ becomes constant for $B>B_{c 2}(\theta)$.

${ }^{18}$ T. Klein, C. Marcenat, L. Lyard, J. Marcus, B. Kang, H.-J. Kim, H.-S. Lee, and S.-I. Lee, in 24th International Conference on Low Temperature Physics, edited by Y. Takano et al., AIP Conf. Proc. No. 850 (AIP, Melville, NY, 2006), p. 595.

${ }^{19}$ See, for instance, K. D. Belashchenko, M. van Schilfgaarde, and V. P. Antropov, Phys. Rev. B 64, 092503 (2001); Y. Kong, O. V. Dolgov, O. Jepsen, and O. K. Andersen, ibid. 64, 020501(R) (2001); A. Brinkman, A. A. Golubov, H. Rogalla, O. V. Dolgov, J. Kortus, Y. Kong, O. Jepsen, and O. K. Andersen, ibid. 65, 180517(R) (2002).

${ }^{20}$ M. E. Zhitomirsky and V.-H. Dao, Phys. Rev. B 69, 054508 (2004).

${ }^{21}$ M. Putti, V. Braccini, C. Ferdeghini, I. Pallecchi, A. S. Siri, F. Gatti, P. Manfrinetti, and A. Palenzona, Phys. Rev. B 70, 052509 (2004). 